\title{
Predicting antidepressant response
}

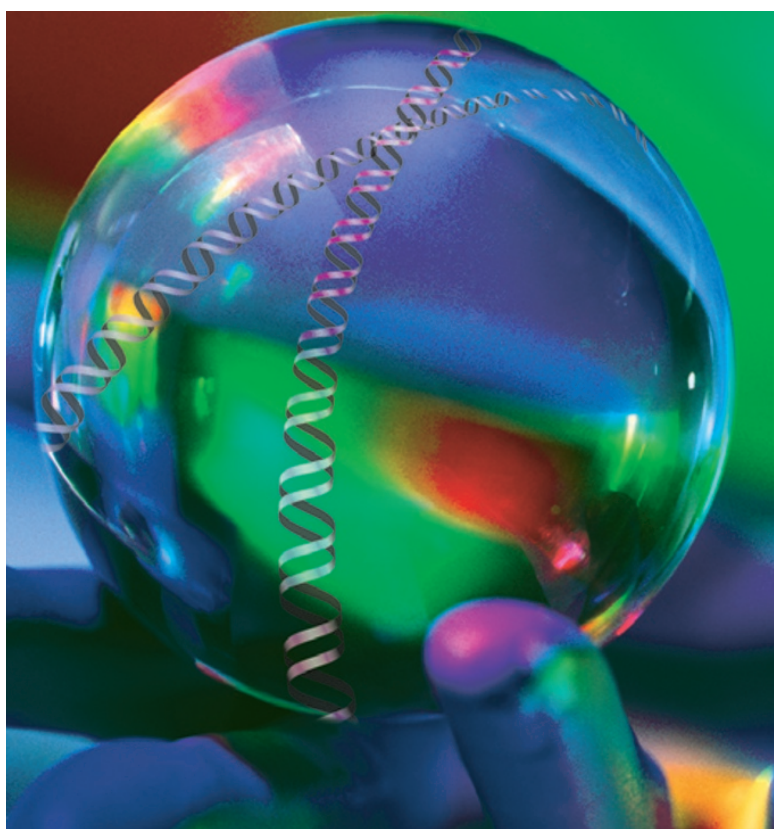

A growing number of single nucleotide polymorphism (SNP)-mapping and genome-wide association (GWA) studies are identifying common genetic variants that are associated with complex diseases such as diabetes, cancer and mood disorders. Such studies can also be used to understand the variability in medication response. In Neuron, Florian Holsboer and colleagues report an important example: genetic variation in the $A B C B 1$ gene can account for some of the differences in antidepressant efficacy.

Overall, only a third of patients on antidepressant medication achieve full remission. A possible reason might be that insufficient drug concentrations reach the nervous system owing to an inadequate ability to cross the blood-brain barrier. Transport molecules on the endothelial cells that line cerebral capillaries, such as P-glycoprotein (P-gp; encoded by $A B C B 1$ ), determine the intracerebral concentration of certain drugs and thus, could be critical for the clinical response to CNS-targeted drugs.

Using mice in which the homologue of the human $A B C B 1$ gene had been deleted, the authors showed that, after administration for 11 days, the brain concentrations of two structurally distinct antidepressants - citalopram (a selective serotonin re-uptake inhibitor) and venlafaxine (a selective serotonin and noradrenaline re-uptake inhibitor) - were up to four-times higher than in wildtype mice. No differences were found for mirtazapine, indicating that citalopram and venlafaxine are P-gp substrates.

Next, they investigated whether functionally relevant genetic variants in $A B C B 1$ in humans could also affect antidepressant concentrations in the brain. From a group of 443 patients receiving antidepressants, they found a highly significant association between 11 SNPs of $A B C B 1$ and remission in patients taking antidepressants that are substrates of P-gp. No such association was found in patients taking mirtazapine (a non-P-gp substrate).

The occurrence of these SNPs varies considerably between ethnic groups, which could explain the differences in therapeutic outcome that are often observed between African Americans and Caucasians receiving antidepressant medication.

Taking a patient's P-gp genetic status into consideration is likely to be important not only for predicting the response to certain antidepressants, but also to many other clinically important drugs (and steroid hormones) that rely on P-gp to penetrate the brain. Moreover, consideration of $A B C B 1$ polymorphisms in the design of clinical trials of P-gp substrates might help stratify patient populations and therefore reduce the cost of developing ineffective treatments.

Monica Hoyos Flight

ORIGINAL RESEARCH PAPER Uhr, M. et al. Polymorphisms in the drug transporter gene $A B C B 1$ predict antidepressant response in depression. Neuron 57, 203-209 (2008) 